\title{
A necklace of Wulff shapes
}

\author{
Joël De Coninck ${ }^{(1)}$, François Dunlop ${ }^{(2)}$, Thierry Huillet ${ }^{(2)}$
}

\begin{abstract}
In a probabilistic model of a film over a disordered substrate, Monte-Carlo simulations show that the film hangs from peaks of the substrate. The film profile is well approximated by a necklace of Wulff shapes. Such a necklace can be obtained as the infimum of a collection of Wulff shapes resting on the substrate. When the random substrate is given by iid heights with exponential distribution, we prove estimates on the probability density of the resulting peaks, at small density.
\end{abstract}

KEYWORDS: Interfaces, random substrate, Wulff shape, SOS model

AMS subject classification: 60K35, 60K37, 82B24, 82B41

\section{Introduction}

A problem in the science of coating is to characterize the surface of a coated material as function of the substrate surface and properties of the coating material (polymer, resin, metal...). The topography of a substrate has an important influence on the properties of the considered material in terms of lubrication, optical properties, wetting... Moreover, it is often desirable to coat this substrate with a thin film to protect the material. Of course, the topography of the thin film surface and of the substrate do not have to be the same. This will be a function of the film thickness.

A statistical mechanical model of a film requires at least two parameters, one associated with a surface tension, the other with a pressure difference or chemical potential controlling the film thickness. The version of the Solid-On-Solid model introduced by Abraham and Smith [AS1, AS2] is the simplest such model. In Section 2, we use it first to model a disordered substrate and then, with a different set of parameters, to model a film on top of the substrate. Numerical simulations show that typical configurations look like a necklace of Wulff shapes suspended from the peaks of the substrate. This motivates Section 3, where only the substrate is random, and the film is defined as the infimum of a collection of Wulff shapes over the substrate. In Section 4 we prove estimates on the density of relevant substrate peaks, when the pressure difference goes to zero. In section 5 we give a Gibbs formulation for the probability density of substrate peak localizations and heights.

\section{Solid-On-Solid film over quenched Solid-On-Solid substrate}

The substrate is a one-dimensional Solid-On-Solid model with Hamitonian

$$
H_{1}=J_{1} \sum_{|i-j|=1}\left|h_{i}^{1}-h_{j}^{1}\right|+K_{1} \sum h_{i}^{1}
$$

(1) Centre de Recherche en Modélisation Moléculaire, Université de Mons-Hainaut, 20 Place du Parc, 7000 Mons, Belgium. Email: Joel.De.Coninck@crmm.umh.ac.be

(2) Laboratoire de Physique Théorique et Modélisation (CNRS - UMR 8089), Université de CergyPontoise, 95302 Cergy-Pontoise, France. Email: dunlop@ptm.u-cergy.fr, huillet@ptm.u-cergy.fr 
where $h_{i}^{1} \in \mathbb{R}_{+}$is the height of the substrate surface at point $i \in \mathbb{Z}$. It can also represent the height of a first coating, fixed before the later deposit of a film.

The substrate being generated according to the Gibbs measure with Hamiltonian (2.1) at temperature $k T=1$, and then quenched, a film is deposited and thermalized according to the Gibbs measure with Hamiltonian

$$
H_{2}=J_{2} \sum_{|i-j|=1}\left|h_{i}^{2}-h_{j}^{2}\right|+K_{2} \sum h_{i}^{2}
$$

at temperature $k T=1$. The film height $h_{i}^{2} \in \mathbb{R}_{+}$at point $i$ obeys $h_{i}^{2} \geq h_{i}^{1}$. This is a grand canonical ensemble where the film volume is controlled by $K_{2}$ while being allowed to fluctuate. In the thermodynamic limit, the properties will be the same as if obtained from a fixed volume ensemble where $\sum\left(h_{i}^{2}-h_{i}^{1}\right)$ is fixed.

The resulting model of a film on a quenched substrate is studied by Monte-Carlo simulation with periodic boundary conditions and a heat bath algorithm. Fig. 1 shows one substrate $h^{1}$ and, on top, thermal averaged films $\bar{h}^{2}$ at various values of the pressure $K_{2}$.

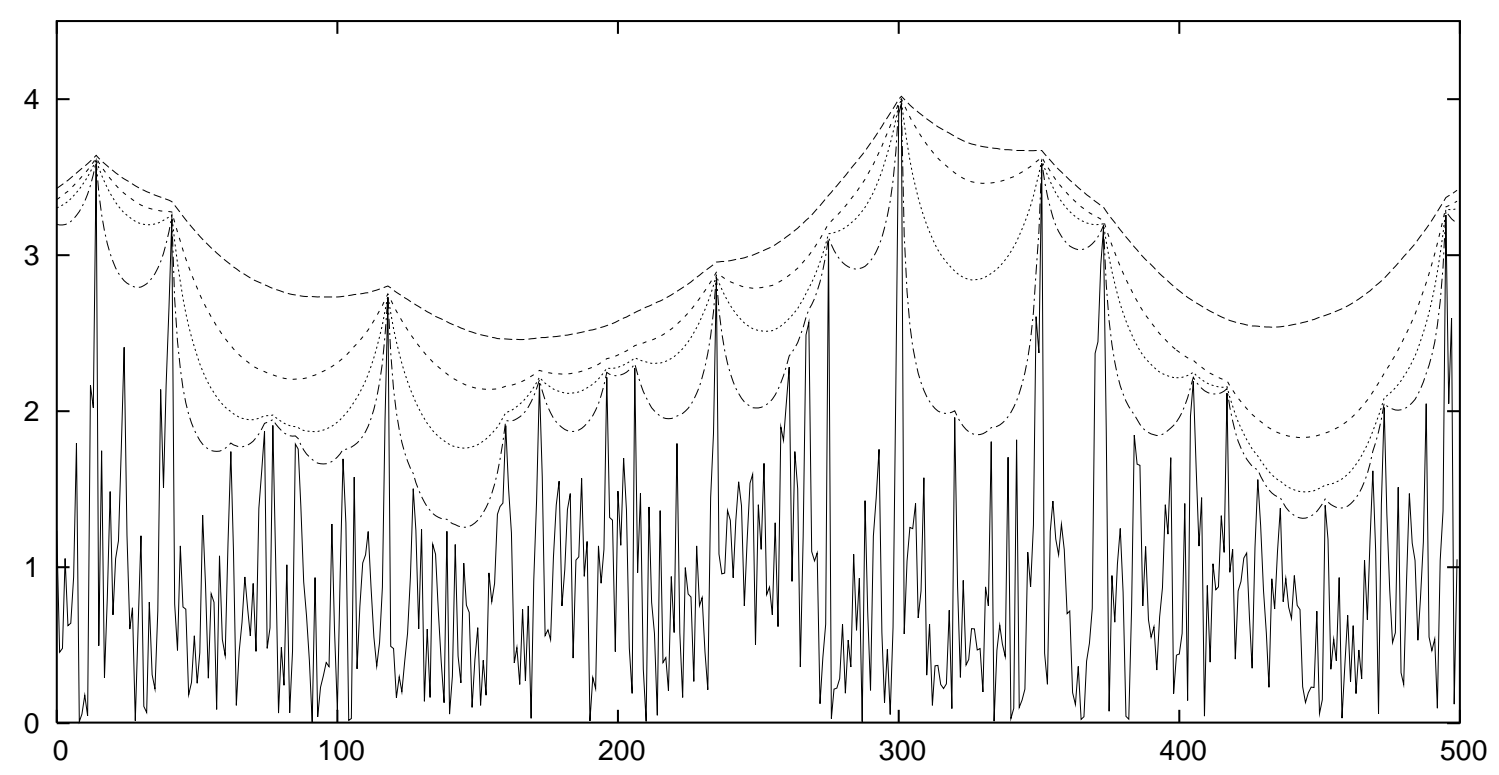

Fig. 1: Substrate $J_{1}=1, K_{1}=0.5$; film $J_{2}=30$ and from top to bottom $K_{2}=0.2,0.5,1,2$.

For small enough $K_{2} / J_{2}$, the film appears to hang as from a set of telegraph poles of random heights. Between two successive poles, the film profile can be checked to be very near a Wulff shape associated with $(2.2)$ at the corresponding value of $K_{2}$. Indeed Fig. 2 shows a portion of substrate, a thermal averaged film with $J_{2}=5$ and $K_{2}=0.125$, and a necklace of Wulff shapes: Each piece is a translate of one and same Wulff shape, with the same $J_{2}$ and $K_{2}$ as the film. The parametric equations of the Wulff shape are [BN,DD]:

$$
\begin{aligned}
& x(\tan \theta)=-\frac{1}{K_{2}} \frac{d}{d(\tan \theta)} \tilde{\sigma}(\tan \theta) \\
& z(\tan \theta)=-\frac{1}{K_{2}}\left(\tilde{\sigma}(\tan \theta)-\tan \theta \frac{d}{d(\tan \theta)} \tilde{\sigma}(\tan \theta)\right)
\end{aligned}
$$


where $\tilde{\sigma}(\tan \theta)$ is the projected surface tension, which for the Solid-On-Solid model takes the form $[\mathrm{DD}]$

$$
\tilde{\sigma}(\tan \theta)=f(\tan \theta)-\log \left(\frac{f(\tan \theta)+2}{J_{2}}\right)
$$

with

$$
f(\tan \theta)=\left(1+\left(J_{2} \tan \theta\right)^{2}\right)^{1 / 2}-1
$$

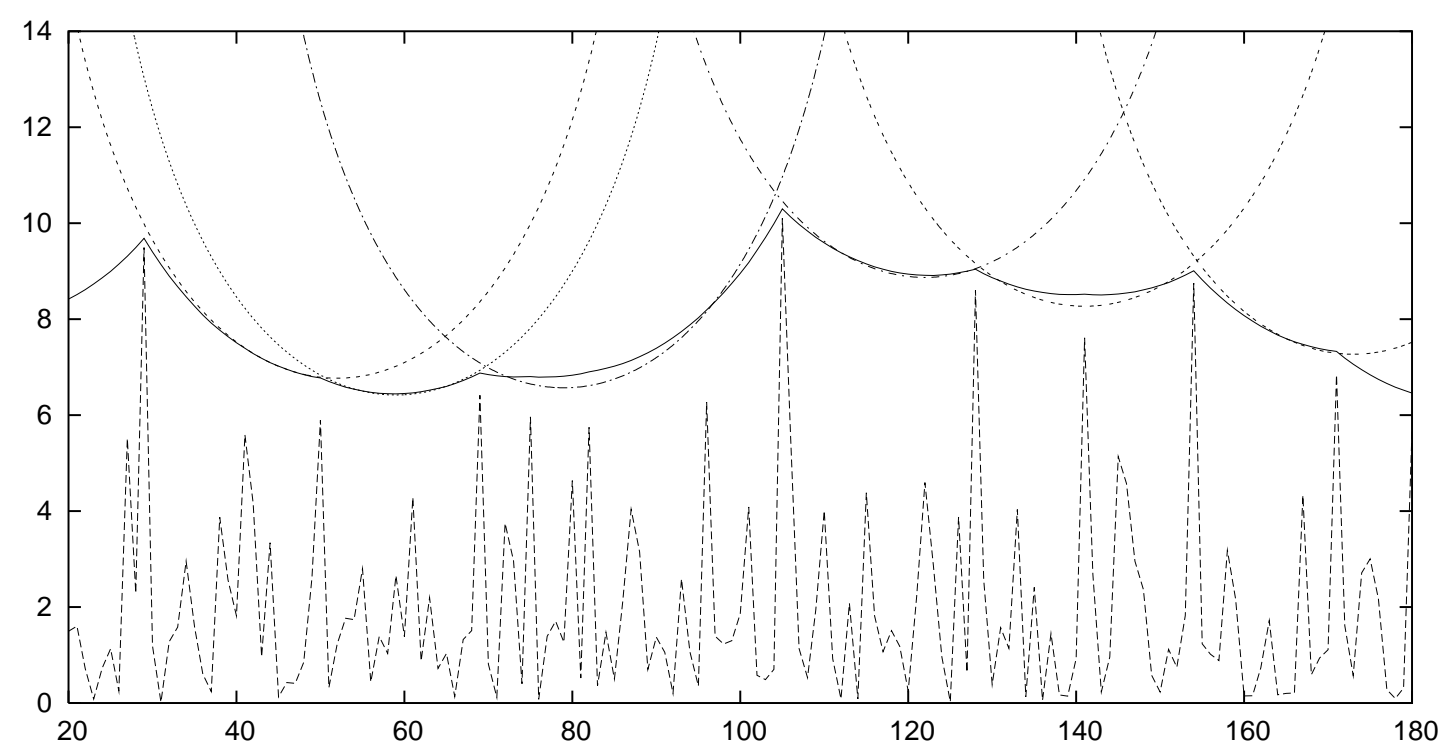

Fig. 2: Substrate $J_{1}=0, K_{1}=0.5$; film (solid line) and Wulff shapes $J_{2}=5, K_{2}=0.125$.

The Wulff necklace is clearly a good approximation, except when the substrate rises near but below the film surface, like near $x \simeq 80$ or $x \simeq 140$ on Fig. 2 , which causes some entropic repulsion. As $K_{2}$ decreases, the number of substrate peaks visible from the look of the film decreases (see Fig. 1). The relevant peaks are large deviations events of the substrate disorder. But there is no obvious a priori criterion to tell which $h_{i}^{1}$ will emerge, depending upon $K_{2}$. The film tension leads to correlations between the poles.

In the following sections, we simplify the model in order to understand the selection by the film of substrate peaks as $K_{2}$ varies. When the substrate has short range correlations, its correlation length may be taken as a basic unit of length. A natural simplification is to assume that it is also the lattice unit, and that the substrate is i.i.d. on this scale: $J_{1}=0$. Then, by scaling the unit of height, there is no loss of generality in taking $K_{1}=1$, leaving two independent parameters $J_{2}$ and $K_{2}$.

The second and main simplification is to consider that when a Wulff shape between two peaks hangs strictly above the substrate between the two peaks, then it is not affected by the substrate between the two peaks. Some fluctuation effects (entropic repulsion) are neglected here, an approximation which is better justified when $J_{2}$ is large.

Our aim will be to estimate the density of relevant peaks as function of $K_{2}$, with different kinds of Wulff shapes. And further to get an idea of the probability distribution of peak localizations and heights. 


\section{A necklace of Wulff shapes}

Let $h_{i}, i \in \mathbb{Z}$ be an iid sequence of exponentially distributed random variables of mean 1 , and let

$$
\mathcal{S}=\left\{(i, z) \in \mathbb{Z} \times \mathbb{R}: z \leq h_{i}\right\}
$$

be the random set representing the substrate. The upper index 1 for the substrate height $h_{i}^{1} \equiv h_{i}$ is now omitted.

Let $1<a \leq \infty$ and let $W:]-a, a[\rightarrow \mathbb{R}$ be a continuous even function, strictly increasing on $\left[0, a\left[\right.\right.$, with $W(0)=0$. If $a=\infty$, we require $W(x)>-b+\lambda|x|^{\alpha}$ for some $b, \lambda, \alpha>0$, for all $x$. Examples:

$$
\begin{aligned}
\text { Cone : } & & W(x)=\lambda|x| \\
\text { Parabola : } & & W(x)=\lambda x^{2} \\
\text { Semi }- \text { circle : } & & W(x)=\lambda^{-1}-\sqrt{\lambda^{-2}-x^{2}} \\
\text { SOS Wulff shape }(z(x)=(2.3)): & & W(x)=z(x)-\frac{1}{K_{2}} \log \frac{2}{J_{2}}
\end{aligned}
$$

with $a=\lambda^{-1}$ for the circle and $a=J_{2} / K_{2}$ for the SOS Wulff shape. Translating the graph of such a function by $\left(x_{*}, h_{*}\right) \in \mathbb{R}^{2}$ will define $W\left(x_{*}, h_{*} ; \cdot\right)$, so that $W(0,0 ; \cdot)=W(\cdot)$ and

$$
W\left(x_{*}, h_{*} ; x\right)=h_{*}+W\left(x-x_{*}\right)
$$

The graph of this function, as a subset of $\mathbb{R}^{2}$, is denoted $W\left(x_{*}, h_{*}\right)$. The film over the substrate (or the coating of the substrate) is then defined almost surely as the graph $I$ of the function $I(x)$ defined in the following proposition:

Proposition 3.1: Under the hypotheses on $W(\cdot)$ stated before (3.2), let

$$
I(x)=\inf \left\{W\left(x_{*}, h_{*} ; x\right):\left(x_{*}, h_{*}\right) \in \mathbb{R}^{2},\left|x_{*}-x\right|<a, W\left(x_{*}, h_{*}\right) \cap \mathcal{S}=\emptyset\right\}
$$

whenever the infimum is taken over a non-empty family, and $I(x)=+\infty$ if $W\left(x_{*}, h_{*}\right) \cap \mathcal{S} \neq$ $\emptyset \forall\left(x_{*}, h_{*}\right)$. Then, almost surely, the infimum is attained and $I(x)<\infty \forall x \in \mathbb{R}$.

Proof. Straightforward. The hypothesis on $W(\cdot)$ could be weakened to $W(x)>-b+$ $\lambda \log |x|$ for suitable $\lambda$. In the following, we shall instead strengthen the hypothesis to make $W$ convex.

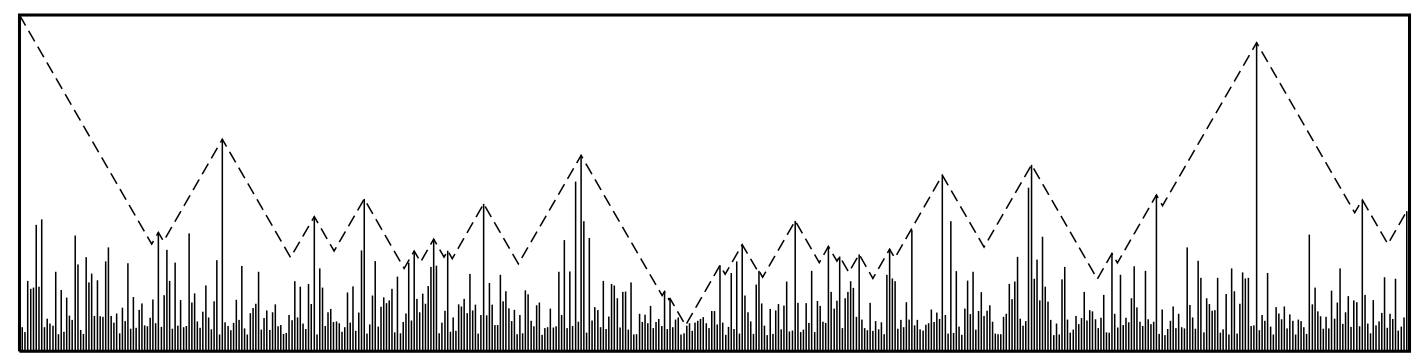

Fig. 3: $I(x)$ with $W(x)=\lambda|x|$. 


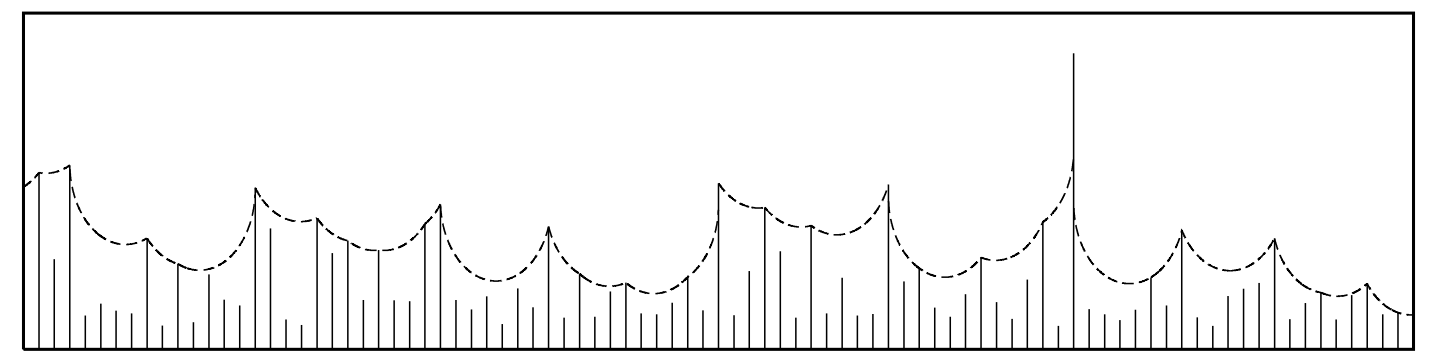

Fig. 4: $I(x)$ with $W(x)$ semi-circular.

In words: We have a "Wulff shape", symmetric about a vertical axis. Above each $x_{*} \in \mathbb{R}$, a "Wulff shape" is taken down from $+\infty$, until it touches the substrate. The film is the infimum of the resulting collection of Wulff shapes. The film height $I(j)$ at an integer point $j$ models the thermal average of $h_{j}^{2}$ in the preceding section.

A Wulff shape in one dimension is a solution to the second order differential equation for the function $W(x)$,

$$
\frac{d}{d x} \frac{d}{d(d W / d x)} \tilde{\sigma}(d W / d x)=K_{2}
$$

where $\tilde{\sigma}(\cdot)$ is the projected surface tension, or interface free energy per unit length of interface projected onto the $x$-axis, as function of the slope $d W / d x$. The parameter $K_{2}$, conjugate to the film volume $\sum h_{i}^{2}$ in $(2.2)$, is the pressure, or pressure difference with the medium above, $\Delta p$. The surface tension should have convexity properties such that the solution $W(x)$ to (3.5) is a convex function, in fact typically strictly convex, which we assume henceforth, except for the special case $W(x)=\lambda|x|$.

A Hamiltonian of the form $(2.2)$ but quadratic in $\left(h_{i}^{2}-h_{j}^{2}\right)$ gives

$$
\tilde{\sigma}(d W / d x)=J_{2}(d W / d x)^{2}+\text { constant }
$$

so that a solution to (3.5) in this case is $W(x)=\lambda x^{2}$ with $\lambda=K_{2} /\left(4 J_{2}\right)$. Solutions to the Wulff equation (3.5) generally scale as

$$
W_{K_{2}}(x)=K_{2}^{-1} W_{1}\left(K_{2} x\right)
$$

where $W_{1}(x)$ is a solution to (3.5) with $K_{2}=1$. The semi-circular shape in (3.2) follows this scaling, with $\lambda$ proportional to $K_{2}$.

When a Wulff shape is taken down from infinity above $x=0$ until it touches the substrate $\mathcal{S}$, say at $\left(j_{0}, h_{j_{0}}\right)$ with $j_{0} \leq 0$ for definiteness, it stops as

$$
W\left(0, h_{*}(0) ; x\right)=h_{j_{0}}-W\left(j_{0}\right)+W(x)
$$

We can then let it slide to the right by an amount $x_{*}$, keeping contact with $\left(j_{0}, h_{j_{0}}\right)$, as

$$
W\left(x_{*}, h_{*}\left(x_{*}\right) ; x\right)=h_{j_{0}}-W\left(j_{0}-x_{*}\right)+W\left(x-x_{*}\right)
$$


At a given $x>0$, this is a strictly decreasing function of $x_{*}$ so long as $x_{*} \leq x$. It is also a strictly decreasing function of $x_{*}$ for $x_{*}>x$, provided $W(\cdot)$ is a strictly convex function: For $y_{*}>x_{*}$,

$$
\frac{W\left(y_{*}\right)-W\left(y_{*}-x\right)}{x}>\frac{W\left(x_{*}\right)-W\left(x_{*}-x\right)}{x}
$$

So we let the Wulff shape slide to the right keeping contact with $\left(j_{0}, h_{j_{0}}\right)$ until it touches a second point $\left(k_{0}, h_{k_{0}}\right)$ :

$$
h_{k_{0}}-h_{j_{0}}=W\left(k_{0}-x_{*}\right)-W\left(j_{0}-x_{*}\right)
$$

We thus get the unique Wulff shape going through $\left(j_{0}, h_{j_{0}}\right)$ and $\left(k_{0}, h_{k_{0}}\right)$, which we denote $W\left(j_{0}, h_{j_{0}}, k_{0}, h_{k_{0}} ; x\right)$. If the first contact point is $\left(k_{0}, h_{k_{0}}\right)$ with $k_{0} \geq 0$, instead of $\left(j_{0}, h_{j_{0}}\right)$ with $j_{0} \leq 0$, the same construction works symmetrically, sliding to the left. In any case we get a Wulff shape at a minimal height, so that, almost surely,

$$
I(x)=W\left(j_{0}, h_{j_{0}}, k_{0}, h_{k_{0}} ; x\right), \quad j_{0} \leq x \leq k_{0}
$$

The proposition below follows easily:

Proposition 3.2: Let $W: \mathbb{R} \rightarrow \mathbb{R}$ be a continuous even function, strictly convex with $W(0)=0$, or $W(x)=\lambda|x|$ with $\lambda>0$. Then $I(x)$ defined in (3.4)(3.1) is also, almost surely,

$$
I(x)=\sup \left\{W\left(j, h_{j}, k, h_{k} ; x\right): j, k \in \mathbb{Z}, j \leq x \leq k\right\}
$$

Proof. $\quad$ Starting from (3.4), we have $I(x)=W\left(x_{*}, h_{*} ; x\right)$. This must be also $I(x)=$ $W\left(j, h_{j}, k, h_{k} ; x\right)$ for some $j, k$ : Otherwise (3.7-11) would give a smaller $I(x)$. And this $W\left(j, h_{j}, k, h_{k} ; x\right)$ must be the supremum, otherwise it would intersect $\mathcal{S}$ (as shown in more detail in the proof of Lemma 5.1 below).

Remark: For the first two examples in (3.2), we have respectively

$$
\begin{gathered}
W\left(j, h_{j}, k, h_{k} ; x\right)=\max \left\{h_{j}-\lambda(x-j), h_{k}+\lambda(x-k)\right\} \\
W\left(j, h_{j}, k, h_{k} ; x\right)=\lambda(x-j)(x-k)+\frac{k-x}{k-j} h_{j}+\frac{j-x}{j-k} h_{k}
\end{gathered}
$$

The random interface $I(x)$ also defines, as a marginal, a point process of interest:

Proposition 3.3: Under the same hypotheses as in Proposition 3.2, let

$$
\mathbb{B}=I \cap \mathcal{S}=\left\{i \in \mathbb{Z}: I(i)=h_{i}\right\}
$$

Then

$$
\mathbb{B}=\left\{i \in \mathbb{Z}: h_{i} \geq W\left(j, h_{j}, k, h_{k} ; i\right) \quad \forall j<i<k\right\}
$$

and, almost surely, $\mathbb{B}$ can be written as $\mathbb{B}=\left\{b_{n}\right\}_{n \in \mathbb{Z}}$ with $b_{n+1}-b_{n} \geq 1 \forall n$, and $b_{0}=$ $\min \left\{b_{n}: b_{n} \geq 0\right\}$. 
Proof. If $i$ belongs to (3.14) then Proposition 3.2 implies that it belongs also to (3.15). If $i$ belongs to (3.15), then we start from

$$
h_{i}=W\left(i, h_{i} ; i\right) \geq W\left(j, h_{j}, k, h_{k} ; i\right) \quad \forall j<i<k
$$

and slide $W\left(i, h_{i} ; i\right)$ to the right following (3.7-11) until it touches $\mathcal{S}$ at $\left(k, h_{k}\right)$ with $h_{i}=$ $W\left(i, h_{i}, k, h_{k} ; i\right)$. Then Proposition 3.2 implies that $i$ belongs also to (3.14).

The set $\mathbb{B}$ is also the set of points which can be obtained like $j_{0}$ or $k_{0}$ in (3.5)-(3.8), starting from any $x \in \mathbb{R}$, not just $x=0$.

\section{Estimates}

Proposition 4.1: Let $W(x)=\lambda|x|$. Then

$$
\lim _{\lambda \searrow 0} \frac{\mathbb{P}(0 \in \mathbb{B})}{\lambda}=\frac{1}{2}
$$

Proof.

$$
\begin{aligned}
\mathbb{P}(0 \in \mathbb{B}) & =\mathbb{P}\left(h_{0} \geq \max \left\{h_{j}-\lambda(x-j), h_{k}+\lambda(x-k)\right\} \quad \forall j<0<k\right) \\
& =\mathbb{P}\left(h_{0} \geq h_{i}-\lambda|i| \quad \forall i \in \mathbb{Z}_{*}\right) \\
& =\int_{0}^{\infty} d x e^{-x} \prod_{i=1}^{\infty}\left(1-e^{-x-\lambda i}\right)^{2}
\end{aligned}
$$

- Upper bound:

$$
\prod_{i=1}^{\infty}\left(1-e^{-x-\lambda i}\right)^{2}<e^{-2 e^{-x} \sum_{i=1}^{\infty} e^{-\lambda i}}=e^{-2 e^{-x} \frac{e^{-\lambda}}{1-e^{-\lambda}}}
$$

so that

$$
\mathbb{P}(0 \in \mathbb{B})<\int_{0}^{\infty} d x e^{-x} e^{-2 e^{-x} \frac{e^{-\lambda}}{1-e^{-\lambda}}}=\frac{1-e^{-\lambda}}{2 e^{-\lambda}}\left(1-e^{-2 \frac{e^{-\lambda}}{1-e^{-\lambda}}}\right)
$$

- Lower bound: For $x>1$, we can use $1-\epsilon>e^{-\epsilon-\epsilon^{2}}$, with $\epsilon=e^{-x-\lambda i}$. Then

$$
\prod_{i=1}^{\infty}\left(1-e^{-x-\lambda i}\right)^{2}>e^{-2 e^{-x} \sum_{i=1}^{\infty} e^{-\lambda i}-2 e^{-2 x} \sum_{i=1}^{\infty} e^{-2 \lambda i}}=e^{-2 e^{-x} \frac{e^{-\lambda}}{1-e^{-\lambda}}-2 e^{-2 x} \frac{e^{-2 \lambda}}{1-e^{-2 \lambda}}}
$$

so that

$$
\begin{aligned}
\mathbb{P}(0 \in \mathbb{B}) & >\int_{1}^{\infty} d x e^{-x} e^{-2 e^{-x} \frac{e^{-\lambda}}{1-e^{-\lambda}}-2 e^{-2 x} \frac{e^{-2 \lambda}}{1-e^{-2 \lambda}}} \\
& >\int_{0}^{\lambda \ln \left(\lambda^{-1}\right)} d y e^{-2 y \frac{e^{-\lambda}}{1-e^{-\lambda}}-2 y^{2} \frac{e^{-2 \lambda}}{1-e^{-2 \lambda}}} \\
& >\frac{1-e^{-\lambda}}{2 e^{-\lambda}}\left(1-e^{-2 \lambda \ln \left(\lambda^{-1}\right) \frac{e^{-\lambda}}{1-e^{-\lambda}}}\right) e^{-2\left(\lambda \ln \left(\lambda^{-1}\right)\right)^{2} \frac{e^{-2 \lambda}}{1-e^{-2 \lambda}}}
\end{aligned}
$$


The upper and lower bounds together imply (4.1).

Proposition 4.2: Let $W(x)=\lambda x^{2}$. Then $\exists a, b>0$ such that $\forall 0<\lambda<1 / 4$

$$
a \frac{\lambda}{\ln \left(\lambda^{-1}\right)}<\mathbb{P}(0 \in \mathbb{B})<3 \sqrt{\frac{\lambda}{\pi}}+b \lambda
$$

Remark: In view of the proof of the upper bound, see below, we expect that $\mathbb{P}(0 \in \mathbb{B})$ is of order $\sqrt{\lambda}$ for small $\lambda$.

Proof.

$$
\mathbb{P}(0 \in \mathbb{B})=\mathbb{P}\left(h_{0} \geq W\left(-j, h_{-j} ; k, h_{k} ; 0\right) \quad \forall j, k \geq 1\right)
$$

with

$$
W\left(-j, h_{-j} ; k, h_{k} ; 0\right)=\frac{k}{j+k} h_{-j}+\frac{j}{k+j} h_{k}-\lambda j k
$$

- Upper bound:

$$
\begin{aligned}
\mathbb{P}\left(0 \notin \mathbb{B} \mid h_{0}=x\right) & =\mathbb{P}\left(\exists j, k>0: x<W\left(-j, h_{-j} ; k, h_{k} ; 0\right)\right) \\
& >\mathbb{P}\left(\exists j, k>0: h_{-j}>x+\lambda j^{2}, h_{k}>x+\lambda k^{2}\right) \\
& =\left(1-\prod_{j=1}^{\infty} \mathbb{P}\left(h_{-j}>x+\lambda j^{2}\right)\right)^{2}
\end{aligned}
$$

where we used

$$
W\left(-j, x+\lambda j^{2} ; k, x+\lambda k^{2} ; 0\right)>x
$$

and $W(\cdots)$ increasing in $h_{-j}$ and in $h_{k}$. Then

$$
\prod_{j=1}^{\infty} \mathbb{P}\left(h_{-j}>x+\lambda j^{2}\right)=\prod_{j=1}^{\infty}\left(1-e^{-x-\lambda j^{2}}\right)<e^{-e^{-x} \sum_{j=1}^{\infty} e^{-\lambda j^{2}}}<e^{-e^{-x}\left(\frac{1}{2} \sqrt{\frac{\pi}{\lambda}}-1\right)}
$$

so that

$$
\begin{aligned}
\mathbb{P}(0 \in \mathbb{B}) & <\int_{0}^{\infty} d x e^{-x}\left(2 e^{-e^{-x}\left(\frac{1}{2} \sqrt{\frac{\pi}{\lambda}}-1\right)}-e^{-2 e^{-x}\left(\frac{1}{2} \sqrt{\frac{\pi}{\lambda}}-1\right)}\right) \\
& =\int_{0}^{1} d y\left(2 e^{-y\left(\frac{1}{2} \sqrt{\frac{\pi}{\lambda}}-1\right)}-e^{-2 y\left(\frac{1}{2} \sqrt{\frac{\pi}{\lambda}}-1\right)}\right) \\
& =3 \sqrt{\frac{\lambda}{\pi}} \frac{1+\frac{1}{3} e^{2-\sqrt{\frac{\pi}{\lambda}}}}{1-2 \sqrt{\frac{\lambda}{\pi}}} \quad \text { for } \lambda<\pi / 4
\end{aligned}
$$

- Lower bound: From the Harris-FKG inequality [H,FKG],

$$
\mathbb{P}\left(\forall j, k>0: x \geq W\left(-j, h_{-j} ; k, h_{k} ; 0\right)\right) \geq \prod_{j=1}^{\infty} \mathbb{P}\left(\forall k>0: x \geq W\left(-j, h_{-j} ; k, h_{k} ; 0\right)\right)
$$




$$
\begin{gathered}
\mathbb{P}\left(\forall k>0: x \geq W\left(-j, h_{-j} ; k, h_{k} ; 0\right)\right)=\int_{0}^{\infty} d y e^{-y} \prod_{k=1}^{\infty} \mathbb{P}\left(x \geq W\left(-j, y ; k, h_{k} ; 0\right)\right) \\
>\int_{0}^{x+\lambda j^{2}} d y e^{-y} \prod_{k=1}^{\infty}\left(1-e^{-\lambda k^{2}-\frac{k}{j}\left(x+\lambda j^{2}-y\right)-x}\right) \\
>\int_{0}^{x+\lambda j^{2}} d y \exp \left(-y-\sum_{k=1}^{\infty} e^{-\lambda k^{2}-\frac{k}{j}\left(x+\lambda j^{2}-y\right)-x}-\sum_{k=1}^{\infty} e^{-2 \lambda k^{2}-\frac{2 k}{j}\left(x+\lambda j^{2}-y\right)-2 x}\right)
\end{gathered}
$$

where we used $1-\epsilon>e^{-\epsilon-\epsilon^{2}}$, with $\epsilon<e^{-x}$ and $x \geq 1$ henceforth. Then

$$
\begin{aligned}
\sum_{k=1}^{\infty} e^{-\lambda k^{2}-\frac{k}{j}\left(x+\lambda j^{2}-y\right)-x}<\sum_{k=1}^{\infty} e^{-\frac{k}{j}\left(x+\lambda j^{2}-y\right)-x}=\frac{e^{-x}}{e^{\frac{x+\lambda j^{2}-y}{j}}-1}<\frac{j e^{-x}}{x+\lambda j^{2}-y} \\
\mathbb{P}\left(\forall k>0: x \geq W\left(-j, h_{-j} ; k, h_{k} ; 0\right)\right)>\int_{0}^{\frac{x+\lambda j^{2}}{2}} d y e^{-y-\frac{j e^{-x}}{x+\lambda j^{2}-y}} \\
>\frac{e^{-\frac{j e^{-x}}{x+\lambda j^{2}}}}{1+\frac{2 j e^{-x}}{\left(x+\lambda j^{2}\right)^{2}}}\left(1-e^{-\frac{x+\lambda j^{2}}{2}-\frac{j e^{-x}}{x+\lambda j^{2}}}\right) \\
>\exp \left(-\frac{j e^{-x}}{x+\lambda j^{2}}-\frac{2 j e^{-x}}{\left(x+\lambda j^{2}\right)^{2}}-e^{-\frac{x+\lambda j^{2}}{2}-\frac{j e^{-x}}{x+\lambda j^{2}}}-e^{-\left(x+\lambda j^{2}\right)-\frac{2 j e^{-x}}{x+\lambda j^{2}}}\right)
\end{aligned}
$$

where $(1-\alpha y)^{-1}<1+2 \alpha y$ for $0<\alpha y<1 / 2$ has been used before integration over $y$, and then again $1-\epsilon>e^{-\epsilon-\epsilon^{2}}$ and also $1 /(1+X)>e^{-X}$. Now

$$
\begin{array}{r}
\prod_{j=1}^{\lambda^{-1}} \mathbb{P}\left(\forall k>0: x \geq W\left(-j, h_{-j} ; k, h_{k} ; 0\right)\right)>\exp \left(-\frac{e^{-x}}{2 \lambda} \ln \frac{x+\lambda^{-1}}{x+\lambda}-\frac{e^{-x}}{2 \sqrt{\lambda x}}\right. \\
\left.-\frac{e^{-x}}{2 \lambda(x+\lambda)}-\frac{e^{-x}}{\sqrt{\lambda x^{3}}}-\sqrt{\frac{2 \pi}{\lambda}} e^{-x / 2}-\sqrt{\frac{\pi}{\lambda}} e^{-x}-2\right)
\end{array}
$$

The sums over $j \in \mathbb{Z}_{+}$in the exponential were bounded by corresponding integrals over $\left[1, \lambda^{-1}\right]$ or $\mathbb{R}_{+}$plus a bound of the maximum of the integrand. Then with $\lambda<1 / 4$ and $x>4$,

$$
\prod_{j=1}^{\lambda^{-1}} \mathbb{P}\left(\forall k>0: x \geq W\left(-j, h_{-j} ; k, h_{k} ; 0\right)\right)>\exp \left(-\frac{e^{-x}}{2 \lambda} \ln \lambda^{-1}-\frac{e^{-x}}{\lambda}-\frac{\sqrt{2 \pi} e^{-\frac{x}{2}}}{\sqrt{\lambda}}-2\right)
$$

For $j>\lambda^{-1}$, the range of integration in (4.15) is chosen as $0<y<x+\lambda j^{2}-j \ln 2$. Then only the first few $k=1,2, \ldots$ play a role for the event $\exists k: x<W\left(-j, y ; k, h_{k} ; 0\right)$. Precisely:

$$
\sum_{k=1}^{\infty} e^{-\lambda k^{2}-\frac{k}{j}\left(x+\lambda j^{2}-y\right)-x}<\frac{e^{-x}}{e^{\frac{x+\lambda j^{2}-y}{j}}-1}<e^{-x-\frac{x+\lambda j^{2}-y}{j}}+2 e^{-x-2 \frac{x+\lambda j^{2}-y}{j}}
$$




$$
\begin{aligned}
\int_{0}^{x+\lambda j^{2}-j \ln 2} d y & \exp \left(-y-e^{-x-\frac{x+\lambda j^{2}-y}{j}}-2 e^{-x-2 \frac{x+\lambda j^{2}-y}{j}}\right) \\
& >\int_{0}^{x+\lambda j^{2}-j \ln 2} d y e^{-y}\left(1-3 e^{-x-\frac{x+\lambda j^{2}-y}{j}}\right) \\
& =1-e^{-\left(x+\lambda j^{2}-j \ln 2\right)}-3 \frac{e^{-x-\frac{x+\lambda j^{2}}{j}}}{1-1 / j}\left(1-e^{-\left(x+\lambda j^{2}-j \ln 2\right)(1-1 / j)}\right) \\
& >1-5 e^{-x-\lambda j}>\exp \left(-5 e^{-x-\lambda j}-25 e^{-2 x-2 \lambda j}\right)
\end{aligned}
$$

for $\lambda<1 / 4$ and $x>2$. Then

$$
\begin{aligned}
\prod_{j>\lambda^{-1}} \mathbb{P}\left(\forall k>0: x \geq W\left(-j, h_{-j} ; k, h_{k} ; 0\right)\right) & >\prod_{j>\lambda^{-1}} \exp \left(-5 e^{-x-\lambda j}-25 e^{-2 x-2 \lambda j}\right) \\
& \geq \exp \left(-\frac{5 e^{-x-1}}{1-e^{-\lambda}}-\frac{25 e^{-2 x-2}}{1-e^{-2 \lambda}}\right)
\end{aligned}
$$

Putting together (4.19) and (4.22) and integrating with $d\left(e^{-x}\right)$ over an interval

$$
\alpha \frac{\lambda}{\ln \left(\lambda^{-1}\right)}<e^{-x}<\beta \frac{\lambda}{\ln \left(\lambda^{-1}\right)}
$$

yields the lower bound and completes the proof of the proposition.

\section{Gibbs measure}

So far the equivalent definitions of $\mathbb{B}$, through (3.14) or (3.15), require a knowledge of the whole system in order to decide whether a point $i \in \mathbb{B}$. Here we will define local Gibbs factors where $\left(b_{n}, h_{b_{n}}\right)$ is coupled to $\left(b_{n-1}, h_{b_{n-1}}\right)$ and $\left(b_{n+1}, h_{b_{n+1}}\right)$ only. We first derive an equivalent definition of $\mathbb{B}$ :

Proposition 5.1: $\mathbb{B}$ defined in Proposition (3.3) is almost surely the only countable ordered subset of $\mathbb{Z}$, denoted $\mathbb{B}=\left\{b_{n}\right\}_{n \in \mathbb{Z}}$, with $b_{0}=\min \left\{b_{n} \geq 0\right\}$, obeying the following two conditions:

$$
\begin{aligned}
& h_{i}<W\left(b_{n}, h_{b_{n}}, b_{n+1}, h_{b_{n+1}} ; i\right) \quad \forall \quad b_{n}<i<b_{n+1} \\
& h_{b_{n}} \geq W\left(b_{n-1}, h_{b_{n-1}}, b_{n+1}, h_{b_{n+1}} ; b_{n}\right) \quad \forall n \in \mathbb{Z}
\end{aligned}
$$

$\mathbb{B}$ is the minimal subset of $\mathbb{Z}$ such that the collection of Wulff shapes suspended from this subset lies above the rest of the substrate, i.e. the minimal subset of $\mathbb{Z}$ satisfying (5.1).

Proof. From Propositions (3.2) and (3.3), $\mathbb{B}$ is almost surely the only countable ordered subset of $\mathbb{Z}$ satisfying (5.1) and

$$
h_{b_{n}} \geq W\left(b_{n-p}, h_{b_{n-p}}, b_{n+q}, h_{b_{n+q}} ; b_{n}\right), \quad \forall n \in \mathbb{Z}, p \geq 1, q \geq 1
$$

We only need to prove that (5.2) implies (5.2'). Let us assume two instances of (5.2),

$$
h_{b_{1}} \geq W\left(h_{b_{0}}, h_{b_{2}} ; b_{1}\right)
$$




$$
h_{b_{2}} \geq W\left(h_{b_{1}}, h_{b_{3}} ; b_{2}\right)
$$

and, contradicting (5.2') with $p=2, q=1$,

$$
h_{b_{2}}<W\left(h_{b_{0}}, h_{b_{3}} ; b_{2}\right) \quad ? ? ?
$$

Let us show that $(5.3 a-b)$ with the absurd $\left(5.3^{\prime}\right)$ would imply that $W\left(h_{b_{1}}, h_{b_{3}} ; \cdot\right)$ and $W\left(h_{b_{0}}, h_{b_{2}} ; \cdot\right)$ have two intersections. Indeed

$$
\begin{array}{rlr}
W\left(h_{b_{1}}, h_{b_{3}} ; b_{1}\right) & \geq W\left(h_{b_{0}}, h_{b_{2}} ; b_{1}\right) & \text { using }(5.3 a) \\
W\left(h_{b_{1}}, h_{b_{3}} ; b_{2}\right) & \leq W\left(h_{b_{0}}, h_{b_{2}} ; b_{2}\right) & \text { using }(5.3 b) \\
W\left(h_{b_{1}}, h_{b_{3}} ; b_{3}\right) & =W\left(h_{b_{0}}, h_{b_{3}} ; b_{3}\right) & \\
& =W\left(h_{b_{0}}, W\left(h_{b_{0}}, h_{b_{3}} ; b_{2}\right) ; b_{3}\right) \\
& \geq W\left(h_{b_{0}}, h_{b_{2}} ; b_{3}\right) & \text { using }\left(5.3^{\prime}\right)
\end{array}
$$

so that $W\left(h_{b_{1}}, h_{b_{3}} ; \cdot\right)$ is higher than $W\left(h_{b_{0}}, h_{b_{2}} ; \cdot\right)$ at $b_{1}$ and $b_{3}$ and lower at $b_{2}$, which implies two intersections, impossible for two Wulff shapes which are translates of one another. Therefore (5.3') cannot hold true; (5.2) with $p=1, q=1$ implies (5.2) also with $p=2, q=1$. The argument extends easily to all $p, q$ and the proof of Proposition (5.1) is readily completed.

Let $l_{n}=b_{n}-b_{n-1}$ and $x_{n}=h_{b_{n}}$. A Gibbs measure formulation for $\left\{l_{n}, x_{n}\right\}_{n \in \mathbb{Z}}$ starts from i.i.d. a priori measures: Counting measure on $\mathbb{Z}_{+}$for each $l_{n}$, exponential distribution $\exp \left(-x_{n}\right) d x_{n}$ on $\mathbb{R}_{+}$for each $x_{n}$. And a product of local Gibbs factors,

$$
\prod_{n} F\left(x_{n}, l_{n+1}, x_{n+1}\right) \prod_{n} G\left(x_{n-1}, l_{n}, x_{n}, l_{n+1}, x_{n+1}\right)
$$

with

$$
F\left(x_{0}, l_{1}, x_{1}\right)=\prod_{i=1}^{l_{1}-1}\left(1-e^{-W\left(0, x_{0}, l_{1}, x_{1} ; i\right)}\right)
$$

and

$$
G\left(x_{-1}, l_{0}, x_{0}, l_{1}, x_{1}\right)=1_{x_{0} \geq W\left(-l_{0}, x_{-1}, l_{1}, x_{1} ; 0\right)}
$$

Let us define explicitly a finite volume Gibbs measure with free boundary conditions:

Proposition 5.2: Let $h_{i}, i \in \mathbb{Z}$ be an iid sequence of exponentially distributed random variables of mean 1 . Let $W: \mathbb{R} \rightarrow \mathbb{R}$ be a continuous even function, strictly convex with $W(0)=0$. Let

$$
I_{[0, L]}(i)=\sup \left\{W\left(j, h_{j}, k, h_{k} ; i\right): 0 \leq j \leq i \leq k \leq L\right\}
$$

and

$$
\mathbb{B}_{[0, L]}=\left\{i \in[0, L] \cap \mathbb{Z}: I_{[0, L]}(i)=h_{i}\right\}=\left\{b_{0}, \ldots, b_{N}\right\}
$$


with $b_{0}=0, b_{N}=L$, and $N \geq 1$. Let $l_{n}=b_{n}-b_{n-1}$ and $x_{n}=h_{b_{n}}$. Then $\left\{N,\left\{l_{1}, \ldots, l_{N}\right\},\left\{x_{0}, \ldots, x_{N}\right\}\right\}$ is distributed according to

$$
\Xi_{[0, L]}^{-1} \prod_{0}^{N} d x_{n} e^{-x_{n}} \prod_{0}^{N-1} F\left(x_{n}, l_{n+1}, x_{n+1}\right) \prod_{1}^{N-1} G\left(x_{n-1}, l_{n}, x_{n}, l_{n+1}, x_{n+1}\right)
$$

where the partition function is

$$
\Xi_{[0, L]}=\sum_{N=1}^{L} \sum_{l_{1} \ldots l_{N}} \int \prod_{0}^{N} d x_{n} e^{-x_{n}} \prod_{0}^{N-1} F\left(x_{n}, l_{n+1}, x_{n+1}\right) \prod_{1}^{N-1} G\left(x_{n-1}, l_{n}, x_{n}, l_{n+1}, x_{n+1}\right)
$$

and the sum over the positive integers $l_{1} \ldots l_{N}$ is constrained by $l_{1}+\ldots+l_{N}=L$.

Proof. Proposition 5.2 is a simple corollary of Proposition 5.1.

Such a model is solvable in principle. A natural first step is to change to a pressure ensemble with $\exp (-p L)$ and $L$ random in order to get rid of the global constraint over $l_{1} \ldots l_{N}$.

Acknowledgments: F. Dunlop acknowledges efficient support and kind hospitality at Université de Mons-Hainaut and CRMM where much of the present work was done.

\section{References}

[AS1] D.B. Abraham, E.R. Smith: Surface film thickening: An exactly solved model, Phys. Rev. B26, 1480-1482, (1982).

[AS2] D.B. Abraham, E.R. Smith: An exactly solved model with a wetting transition, J. Stat. Phys. 43, 621-643 (1986).

[BN] H. van Beijeren, I. Nolden: pp 259-300 in Structure and Dynamics of Surfaces II, edited by W. Schommers and P. von Blanckenhagen. Topics in Current Physics Vol. 43 (Springer-Verlag, Berlin Heidelberg, 1987).

[DD] J. De Coninck, F. Dunlop: Partial to complete wetting: A microscopic derivation of the Young relation, J. Stat. Phys. 47, 827-849 (1987).

[FKG] C. Fortuin, P. Kasteleyn, J. Ginibre: Correlation inequalities on some partially ordered sets, Comm. Math. Phys. 79, 141-151 (1981).

[H] T. E. Harris: A lower bound for the critical probability in a certain percolation process, Proc. Camb. Phil. Soc. 59, 13-20 (1960). 